\title{
How to Share Data Securely
}

\author{
X. Sean Wang \\ School of Computer Science, Fudan University, Shanghai, China \\ xywangCS@fudan. edu.cn
}

Data is increasingly available in a digital form, and data about us is being continuously collected. Such data has made possible many interesting and useful applications, and in essense made it possible for the Web to exist in the current form. Sharing this data makes a lot of sense for many reasons. However, personal privacy has become a concern. In this talk, I will touch upon a recent study of the privacy data leakage problem of mobile apps in China, and discuss various ways to protect user data.

However, completely locking up data is neither desirable nor necessary. How to share our data in a secure way becomes an interesting question. Privacy protection of data has been a research problem for decades, with many interesting results emerging in the last 15 or so years. In this talk, I will discuss a framework for secure data sharing, linking many of the existing solutions under a unified principle. The hope is to develop new insights and better data sharing methods. 